\title{
Movimientos socioterritoriales. Casos de América Latina y Europa
}

\author{
Socioterritorial movements. Cases from Latin America and Europe
}

\author{
Sam Halvorsen \\ s.halvorsen@qmul.ac.uk \\ School of Geography, Queen Mary University of \\ London, Reino Unido \\ Bernardo Mançano Fernandes \\ mancano.fernandes@unesp.br \\ Departamento de Geografia, Universidad Estadual \\ Paulista, Brasil \\ Fernanda Valeria Torres \\ ftorres@fahce.unlp.edu.ar \\ Centro de Investigaciones Socio-Históricas / \\ Instituto de Investigaciones en Humanidades y \\ Ciencias Sociales / Facultad de Humanidades y \\ Ciencias de la Educación / Universidad Nacional de \\ La Plata, Argentina
}

Recepción: 13 Junio 2021

Aprobación: 17 Julio 2021

Publicación: 01 Noviembre 2021

Cita sugerida: Halvorsen, S., Mançano Fernandes, B. y Torres, F.V. (2021). Movimientos socioterritoriales. Casos de América Latina y Europa. Geograficando, 17(2), e097.

https://doi.org/10.24215/2346898Xe097
Resumen: Se desarrolla la noción de "movimientos socioterritoriales” del geógrafo Bernardo Mançano Fernandes, en tanto una categoría analítica específica para aquellos movimientos sociales que tienen como objetivo central la apropiación del espacio en pos de lograr su proyecto político. Se distingue el concepto de movimiento socioterritorial del de movimiento social y del de movimiento socioespacial, y se proponen cuatro ejes: estrategia, identidad, sociabilidad política e instituciones, a través de tres casos de estudio: el MST en Brasil, la Organización Barrial Tupac Amaru en la Argentina y el movimiento "Occupy" en Londres. Se concluye con algunas diferencias claves entre los casos estudiados y se evalúan las potencialidades y límites del análisis socioterritorial.

Palabras clave: Movimientos socioterritoriales, Territorio, MST, Occupy, Argentina.

Abstract: This paper develops Brazilian geographer Bernardo Mançano Fernandes' notion of "socio-territorial movements" as an analytical category for social movements that have as their central objective the appropriation of space in pursuit of their political project. It does so by contrasting the concept of socio-territorial movement to those of social movement and sociospatial movement, and proposing four axes of analysis for socio-territorial movements: strategy, identity, political sociability and institutions. These axes are considered across three case studies: the MST in Brazil; an urban social movement from North-West Argentina called the "Tupac Amaru Neighborhood Organization"; and the 2011 "Occupy" movement in London. In conclusion, we discuss some key differences between the movements studied, and consider the limits to a socio-territorial analysis.

Keywords: Socio-territorial movements, Territory, MST, "Occupy" Movement, Argentina. 


\section{INTRODUCCIÓN}

¿Por qué el territorio es importante para analizar los movimientos sociales? ¿Qué permite comprender? A pesar de la aparente centralidad del territorio -entendido como la apropiación y el control del espacio a través de formas de poder-para comprender los movimientos sociales a lo largo del planeta (ocupación de tierras, activismo indígena, organizaciones barriales, etc.), ha habido una carencia de atención a esta cuestión por parte de la geografía anglosajona, en comparación con la extensa literatura dedicada al lugar y a las políticas contenciosas, la escala y las redes (Miller, 2000; Nicholls, 2009; Nicholls, Miller y Beaumont, 2013; Routledge y Cumbers, 2009). Sólo recientemente los geógrafos anglófonos comenzaron a considerar las prácticas de territorialización y las disputas en torno a la territorialidad, en tanto elementos significativos para el análisis de los movimientos sociales (Agnew y Oslender, 2013; Ince, 2012; Routledge, 2015), en parte inspirados por la bibliografía de Latinoamérica, en la cual el territorio hace mucho tiempo es central para las teorías y las prácticas políticas (Halvorsen, 2019). Dentro de la amplia bibliografía latinoamericana referida al análisis espacial de los movimientos sociales, podemos citar a Lopes de Souza, 1995, 2011; Fernandes, 2000b, 2005; Porto-Gonçalves, 2001; Oslender, 2002; Haesbeart, 2004, 2013; Saquet, 2009; dos Santos, 2011 y Tobío, 2014. El presente artículo retoma las intervenciones recientes y propone un marco conceptual que analice los movimientos sociales a través del eje del territorio, desarrollando la noción del geógrafo brasilero Bernardo Manzano Fernandes de "movimiento socioterritorial", en tanto herramienta analítica aplicada a tres estudios de casos de Argentina, Brasil y el Reino Unido.

Los movimientos socioterritoriales tienen el territorio como su característica definitoria, su raison d'être, sin la cual no existirían (Fernandes, 2005). Desde esta perspectiva, el territorio es entendido como proceso dinámico y relacional, que va más allá de las formas modernas y las relaciones de poder de la soberanía estatal (Elden, 2013). Fernandes, en línea con diversos colegas contemporáneos de América Latina (Schneider y Tartaruga, 2006; Souza, 1995), se basa en un conjunto heterodoxo de teóricos, que incluye a Lefebvre (2013), Raffestin (1980) y Santos (1996), para definir el territorio como "el espacio apropiado por una determinada relación social que lo produce y lo mantiene mediante una forma de poder' (Fernandes, 2005, p. 27). El territorio es multidimensional y multiescalar, e incorpora tanto el espacio material como el inmaterial, y diversas intencionalidades que pueden producir contradicciones, solidaridad y conflictividad (Fernandes, 2005). Los movimientos socioterritoriales existen a través de la apropiación de un espacio, mediante los procesos de territorialización desterritorialización reterritorialización (TDR), para la realización de sus objetivos políticos. El territorio apropiado (por ejemplo, la ocupación de tierras, barrios, ciudad) es, a su vez, el objeto de disputa de los militantes, que buscan conquistar una fracción del territorio de los proyectos políticos dominantes, vinculados al Estado y al capital.

Este artículo operacionaliza el concepto de movimientos socioterritoriales mediante un análisis comparativo, que aspira a extender su uso y a proporcionar un conjunto de herramientas para el trabajo posterior. La comparación es entendida aquí como un medio para generar ideas desde múltiples puntos de vista geográfico-históricos, y configurar un modo de análisis abierto y multiplicador (Hart, 2016; Robinson, 2016). Reconociendo los límites de todos los saberes y la pluralidad, o "ecología", de saberes que coexisten en el mundo (Santos, 2009), presentamos tres estudios de caso diferentes, cada uno orientado en torno a la apropiación política del espacio, para examinar con mayor profundidad algunas de las dimensiones de los movimientos socioterritoriales. Proponemos cuatro ejes para analizar los movimientos socioterritoriales. Es necesario aclarar que muchos otros ejes y dimensiones podrían haber sido relevados y que los que proponemos se superponen y son relacionales; sin embargo, proporcionan un medio para comprender y analizar algunas de las características de los movimientos socioterritoriales.

En primer lugar, el territorio es movilizado ${ }^{1}$ como la principal estrategia de los movimientos para lograr sus objetivos. Mientras muchos de los movimientos sociales se apropian del espacio como una modalidad de lucha (por ejemplo, las sentadas realizadas por activistas de derechos humanos), para los 
movimientos socioterritoriales el territorio es el objeto central de su lucha y es la clave para sus fines y necesidades. En segundo lugar, a partir del territorio se construyen la identidad y la subjetividad política en los movimientos socioterritoriales, y se generan tanto las oportunidades como las limitaciones para su potencial de movilización. En tercer lugar, el territorio es un lugar de encuentro y socialización política que permite a los movimientos generar nuevos valores y nuevas relaciones sociales. Finalmente, a través del proceso de TDR los movimientos sociales institucionalizan sus formas organizacionales, a la vez que se superponen y se enredan ${ }^{2}$ con el proyecto territorial del Estado.

En lo que sigue, desarrollaremos la noción de movimiento socioterritorial contrastándola con las categorías de movimiento social y movimiento socioespacial, describiendo con mayor detalle los cuatro ejes del análisis comparativo. El resto del trabajo analiza los tres estudios de caso: el MST (Movimiento Sin Tierra) en Brasil; un movimiento social urbano de Argentina, la Organización Barrial Tupac Amaru y el movimiento Occupy de 2011 en Londres. En cada caso utilizamos los cuatro ejes propuestos para comprender cómo y por qué se movilizan los movimientos socioterritoriales; y, en el caso de Occupy London, se enfatizan los límites de la categoría socioterritorial. Por último, en las conclusiones consideramos algunas de las diferencias clave entre nuestros tres estudios de caso y las potencialidades y límites de la perspectiva en torno a los movimientos socioterritoriales como una teoría itinerante, que surgió desde América Latina en general y desde el caso del MST en particular.

\section{MOVIMIENTOS SOCIALES, MOVIMIENTOS SOCIOESPACIALES Y MOVIMIENTOS SOCIOTERRITORIALES}

A pesar del creciente interés en las espacialidades, ha habido una falta de atención al territorio en los estudios de los movimientos sociales por parte de la geografía anglófona. Un lugar destacado ha tenido la categoría de redes y la categoría de lugar, pero no así la categoría de territorio, ${ }^{3}$ por lo que en otro trabajo (Halvorsen, Fernandes, Torres, 2019) hemos defendido el análisis socioterritorial como una categoría analítica muy útil para los investigadores anglófonos que estudien la politicidad de base de los movimientos sociales.

La noción de movimiento socioterritorial fue desarrollada por el geógrafo brasileño Fernandes a partir de su larga relación de trabajo y militancia con el MST (Fernandes 2000a; 2000b; 2005; Fernandes y Martin 2004). Basado en pensadores de Latinoamérica y Europa (Harvey, Lefebvre, Raffestin, Santos, Soja), el autor argumenta en pos de la inseparabilidad del espacio y los movimientos sociales, y recategoriza ambas nociones como movimiento socioespacial (Fernandes, 2000a). Mientras todos los movimientos sociales, como cualquier fenómeno social, están necesariamente constituidos en y a través del espacio, en ciertos casos la producción del territorio se vuelve un objetivo central y una característica definitoria. Definimos aquí el territorio como la apropiación del espacio a través de relaciones sociales que lo producen y mantienen mediante relaciones de poder (Fernandes, 2000b), una definición común en la literatura latinoamericana que tiene su antecedente en la recepción de la obra de Raffestin (1980). Estas aproximaciones al concepto de territorio se alejan en buena parte de la literatura latinoamericana, del énfasis en el Estado y su poder soberano (Lopes de Souza, 2001; Fernandes, 2000a, 2005; Porto-Gonçalves, 2001; Haesbaert, 2004; Saquet, 2009, entre otros).

Territorio es un sitio dinámico, producto de conflictos y confrontaciones entre proyectos políticos en competencia que utilizan las prácticas de territorialización, desterritorialización y reterritorialización para alcanzar sus objetivos, tanto en sus formas materiales como inmateriales (Fernandes, 2000b). Los movimientos socioterritoriales no se producen sólo a través de ocupaciones urbanas y/o rurales, sino que incluyen también cualquier iniciativa de apropiación y control de espacios con el objetivo de llevar adelante proyectos políticos. Como indica Fernandes (2005, p. 24): "Los movimientos socioterritoriales construyen espacios políticos para conquistar sus objetivos, se espacializan y promueven otro tipo de territorio". 
Basándonos en esta definición, argumentamos que los movimientos socioterritoriales representan una categoría diferente y con especificidades respecto de las categorías "movimientos sociales" y "movimientos socioespaciales". Los geógrafos ya han acertado en resaltar que todos los movimientos sociales se construyen en y a través del espacio, y sus relaciones sociales necesariamente se desarrollan en formas espaciales (Miller, 2000; Routledge, 2017). También un número importante de críticas y aportes a las teorías de los movimientos sociales ha sido elaborado, iluminando la dimensión espacial de categorías como recursos de movilización, procesos de enmarcado y construcción de identidad (Martin, 2003; Miller, 2000; Nicholls, 2009). En este sentido, todo movimiento social puede ser analizado como un movimiento socioespacial (Fernandes, 2000a). Sin embargo, no todo movimiento social se apropia de un espacio como un objeto central de sus proyectos políticos. Los movimientos sociales pueden generar identidades y fuertes lazos con relación a un lugar y pueden apuntar a centros de poder en diferentes escalas, pero el territorio es a menudo secundario para los objetivos y metas del movimiento. Movimientos LGTB+ o movimientos por los derechos humanos, por ejemplo, pueden tener como su objetivo principal la emancipación, el empoderamiento y reconocimiento de grupos sociales marginados o prevenir el abuso a los derechos humanos en pos de crear sociedades más justas e inclusivas. Para los movimientos socioterritoriales la transformación de relaciones socioespaciales en un espacio demarcado y apropiado es el objetivo central de su movilización, y el territorio, entonces, proporciona su raison d'être. Mientras todos los movimientos socioterritoriales son socioespaciales, no todos los movimientos socioespaciales son socioterritoriales. 
FIGURA 1

\begin{tabular}{|c|c|c|c|}
\hline & $\begin{array}{l}\text { Movimientos } \\
\text { sociales }\end{array}$ & $\begin{array}{l}\text { Movimientos } \\
\text { socioespaciales }\end{array}$ & $\begin{array}{l}\text { Movimientos } \\
\text { socioterritoriales }\end{array}$ \\
\hline Estrategia & $\begin{array}{l}\text { Recursos de } \\
\text { movilización; } \\
\text { estructura de } \\
\text { oportunidades } \\
\text { políticas }\end{array}$ & $\begin{array}{l}\text { Enmarcado en } \\
\text { lugares; } \\
\text { saltos de escala; } \\
\text { redes }\end{array}$ & $\begin{array}{c}\text { Territorios } \\
\text { apropiados en } \\
\text { búsqueda de } \\
\text { proyectos políticos, } \\
\text { para la } \\
\text { supervivencia y la } \\
\text { acción contenciosa }\end{array}$ \\
\hline Identidad & $\begin{array}{c}\text { Identidad colectiva; } \\
\text { cambio social }\end{array}$ & $\begin{array}{c}\text { Sentido de lugar, } \\
\text { cambio } \\
\text { socioespacial }\end{array}$ & $\begin{array}{c}\text { Identidad } \\
\text { construida a través } \\
\text { del territorio, que } \\
\text { produce nuevas } \\
\text { subjetividades } \\
\text { políticas. }\end{array}$ \\
\hline $\begin{array}{l}\text { Socialización } \\
\text { política }\end{array}$ & $\begin{array}{l}\text { Enmarcado para la } \\
\text { contención; } \\
\text { afectos y } \\
\text { emociones }\end{array}$ & $\begin{array}{l}\text { Creación y } \\
\text { producción del } \\
\text { espacio; } \\
\text { relaciones de lazos } \\
\text { fuertes (basadas en } \\
\text { el lugar) y de lazos } \\
\text { débiles (en red) }\end{array}$ & $\begin{array}{l}\text { Encuentros y } \\
\text { valores formados } \\
\text { en el desarrollo del } \\
\text { territorio. }\end{array}$ \\
\hline Institucionalización & $\begin{array}{c}\text { Formas } \\
\text { organizacionales; } \\
\text { autonomía vs. } \\
\text { cooptación }\end{array}$ & $\begin{array}{l}\text { Constitución } \\
\text { escalar (vertical) y } \\
\text { en red (horizontal) } \\
\text { de movimientos }\end{array}$ & $\begin{array}{c}\text { Territorialización / } \\
\text { Desterritorialización } \\
\text { / } \\
\text { Reterritorialización } \\
\text { (TDR) }\end{array}$ \\
\hline
\end{tabular}

Fuente: Elaboración propia

A fin de profundizar el desarrollo de este concepto, proponemos cuatro ejes de análisis para los movimientos socioterritoriales que emergen como cuestiones compartidas en nuestros casos de estudio. Siguiendo a Fernandes entendemos los movimientos socioterritoriales como una categoría multidimensional y relacional. Los cuatro ejes se encuentran fuertemente interrelacionados y no pretenden agotar todos los posibles ejes de análisis. Con el objetivo de brindar mayor claridad al desarrollo del esquema, presentamos un cuadro en el cual se ejemplifican algunas diferencias clave entre las categorías analíticas de movimiento social, movimiento socioespacial y movimiento socioterritorial en función de nuestro análisis de los cuatro ejes (ver Figura 1). Este esquema es propuesto como un punto de partida en el camino de incorporar el concepto de movimiento socioterritorial, del que consideramos que tiene suficiente especificidad conceptual para ser tenido en cuenta y profundizar su desarrollo. Analizamos con mayor detalle los cuatro ejes presentados en las columnas del cuadro, a la vez que destacamos las diferencias de análisis entre movimientos sociales, socioespaciales y socioterritoriales. 
En primer lugar, el territorio es central para las estrategias de los movimientos socioterritoriales. Los estudios sobre movimientos sociales han construido diversas teorías para explicar las estrategias de los movimientos sociales, entre las cuales la teoría de los recursos de movilización y la teoría de las estructuras de oportunidades políticas son las más importantes (McAdam, Tarrow y Tilly, 2005; Tarrow, 1997). Estos enfoques explican la manera mediante la cual los movimientos sociales se movilizan de acuerdo con la disponibilidad interna de recursos (financiamiento, militancia) y con las condiciones institucionales externas que puedan incentivar o desincentivar la acción contenciosa. Un enfoque sobre los movimientos socioespaciales extiende esto, enfatizando la manera en la que la movilización de recursos y la confrontación con los poseedores del poder tiene una espacialidad para ellos. Ello ha sido elaborado con conceptos como "place framing" (Martin, 2003), "scale jumping” (Glassman, 2002) y "networking” (Juris, 2008; Roultledge y Cumbers, 2009). Los movimientos socioterritoriales también se comprometen con múltiples espacialidades de contención, pero tienen como objetivo primordial la apropiación del espacio en pos de las posibilidades de supervivencia y potencialidad de sus proyectos políticos. El territorio es más que el terreno de resistencia ("terrain of resistance": Routledge, 1993) a través del cual los militantes se movilizan para influir con sus demandas en los titulares del poder y también supera el proyecto de soberanía vinculado a la estatalidad moderna (Elden, 2013; Nicholls et. al., 2013). Territorio es el espacio apropiado a través de las prácticas e ideas de cualquier actor en búsqueda de sus proyectos políticos (Raffestin, 1980). Si bien necesariamente se comprometen con territorios dominantes basados en el Estado, los movimientos socioterritoriales están principalmente orientados a transformar las relaciones socioespaciales mediante el control político y la apropiación de un espacio delimitado.

En segundo lugar, los movimientos socioterritoriales desarrollan su subjetividad en y a través del territorio, lo que provee tanto oportunidades como constreñimientos a su potencial de movilización. Las teorías de los nuevos movimientos sociales han identificado la centralidad de la identidad colectiva para la conformación de movimientos sociales, particularmente en el contexto de la sociedad postindustrial, en la cual los clivajes sociales se han estructurado en múltiples dimensiones (género, raza, medioambiente, sexualidad) (Melucci, 1980). En la búsqueda de sus objetivos, las identidades de los movimientos sociales están, a su vez, moldeadas por sus luchas por el cambio social. Los geógrafos y las geógrafas han subrayado que las políticas de identidad están fuertemente influidas por las políticas de lugar y esa identidad está a su vez ligada al cambio socioespacial (Keith y Pile, 1993). El sentido de lugar que informa la identidad no es necesariamente interno o parroquial, sino que puede extenderse en diferencias históricas y geográficas (Massey, 1991). Mientras que los movimientos territoriales también se configuran por sentidos de lugar relacionales, la identidad de sus miembros se transforma a través de la construcción de un territorio, al punto que muchos militantes eligen identificarse explícitamente en relación con un territorio apropiado (como es el caso de los tres estudios de caso analizados en este artículo). Movimientos de trabajadores desocupados que emergieron en las periferias de Buenos Aires en los años 90, por ejemplo, se apropiaron del territorio barrial como un mecanismo de generación de nuevas subjetividades políticas en el contexto del Estado neoliberal y posindustrial (Dinerstein, 2015; Grimson, Ferraudi y Segura, 2009).

En tercer lugar, la socialización política de los movimientos socioterritoriales construye nuevos encuentros y valores en el curso de la movilización del territorio. La teoría del movimiento social ha tendido a enfatizar la importancia de los procesos de "enmarcado" para dar forma a los entendimientos políticos compartidos sobre lo que sucede en el curso de la movilización (McAdam, Tarrow y Tilly, 2005). Más recientemente, algunos académicos han enfatizado la importancia de las emociones para comprender la acción colectiva, la protesta y los movimientos sociales (Scribano, 2008; Poma y Gravante, 2017). Algunos autores, por ejemplo, analizan las ciudades en tanto generadoras de plexos de sensaciones. Los procesos de fragmentación y segregación socio-espacial se combinan con determinadas formas de percibir y sentir la ciudad, y conforman experiencias particulares de lo urbano que configuran sensibilidades diferenciadas y enclasadas del habitar, desde las cuales emerge el conflicto urbano (Cervio y Vergara, 2017). Podemos decir que estas relaciones espaciales tienden 
a estructurar la socialización política de los movimientos sociales a través de la cual se desarrollan valores y normas comunes. Los movimientos socioterritoriales están estructurados por las prácticas de encuentro y los valores que se forman en el desarrollo del territorio, definido como la apropiación política de algún espacio delimitado. La materialidad del espacio apropiado es crucial para generar nuevos encuentros y forjar valores políticos que se construyen en común. El territorio brinda una oportunidad para la socialización política y también estructura los valores políticos que se producen al hacerlo.

Finalmente, los movimientos socioterritoriales crean nuevas instituciones en el proceso de territorialización, desterritorialización y reterritorialización (TDR) de sus proyectos políticos, tanto como de los proyectos de otros. Los estudios sobre movimientos sociales han tendido a prestar atención a formas organizativas particulares (por ejemplo, estructuras jerárquicas o conectivas: Tarrow, 1997) y a los desafíos de constituir movimientos sociales en formas institucionales duraderas, a menudo comprendidas mediante los dilemas de autonomía versus cooptación en términos de su relación con estructuras dominantes como el Estado (Dinerstein, 2015; Reyes, 2012; Sitrin, 2012; Stahler-Sholk, Vanden y Becker, 2014). Está claro que tanto la institucionalización de los movimientos sociales como su relación con otras instituciones (por ejemplo, el Estado) poseen una espacialidad (Nicholls et. al., 2013; Routledge, 2017). Los movimientos socioterritoriales utilizan las prácticas de TDR para crear nuevas formas de organización que puedan tener una resistencia duradera. La territorialización de los movimientos puede, por sí misma, proporcionar los recursos, habilidades y conocimientos necesarios para mantener una forma de organización alternativa (Routledge, 2015). Para algunos autores en América Latina, como Tobío (2010) y Torres (2016), los movimientos sociales generan territorialidades no estatales, sobre todo allí donde el Estado es débil para efectivizar su poder (O’Donnell, 1993). A su vez, esto puede depender de la desterritorialización y el reemplazo de estructuras institucionales preexistentes (por ejemplo, propietarios de tierras en el territorio ocupado) y llevar a la posterior desterritorialización tanto del movimiento como de los proyectos políticos desplazados. En todos los casos, la territorialización de una nueva forma organizativa es altamente visible y necesariamente implicará un compromiso con el Estado: ya sea mediante la dispersión continua del poder (Zibechi, 2006) o mediante la incorporación y reterritorialización dentro de las instituciones estatales (Rossi, 2017).

El resto del documento aplica estos cuatro ejes analíticos a tres estudios de caso que atraviesan contextos geográficos (urbano y rural, norte y sur), y utiliza la comparación como un medio para abrir y ampliar el diálogo entre diferentes experiencias y conocimientos. Entendemos la comparación como un proceso relacional que, al reunir diferentes casos (Hart, 2016), desarrolla ideas sobre la movilización de los movimientos socioterritoriales. Aceptando la parcialidad de todos los sitios de producción de conocimiento (Mignolo, 2000; Santos, 2009), y reconociendo el potencial de establecer relaciones entre casos aparentemente diferentes (Robinson, 2016), esperamos que el análisis presentado aquí estimule un mayor diálogo académico sobre la movilización de territorios respecto de los movimientos sociales en todo el mundo.

\section{Brasil: el “Movimento dos Trabalhadores Rurais Sem Terra” (MST)}

El Movimiento de Trabajadores sin Tierra (MST) es el más importante movimiento socioterritorial de Brasil que lucha por la tierra y la reforma agraria en un país con uno de los más altos niveles de concentración de la tierra del mundo. Entre el año 2000 y el año 2016 el informe de DATALUTA registró 138 movimientos que pelean por la tierra, que movilizan 828.326 familias (o más de tres millones de personas), de las cuales el $64 \%$ estaban organizadas en el MST (Girardi et. al., 2017). Durante el mismo período, 7.049.073 personas participaron en protestas sobre temáticas relacionadas con la lucha por la tierra y la reforma agraria (por ejemplo: crédito agrícola, infraestructura educativa), como marchas, bloqueos de rutas, ocupación de edificios públicos y campamentos. 
La lucha por la tierra llevada adelante por los movimientos socioterritoriales como el MST puede ser entendida como una forma de política pública de base. Las acciones se desarrollan mediante la planificación, las estrategias y los recursos de familias de bajos ingresos, excluidas de la tierra y el trabajo, que buscan transformar sus realidades. Las ocupaciones estratégicas de la tierra han conquistado fracciones de territorios dominantes, lo que obliga al Estado a responder facilitando la creación de asentamientos permanentes en los que los trabajadores producen alimentos, fibra y energía. Estos movimientos han formado la columna vertebral de la reforma agraria en Brasil y han sido impulsados por la movilización de movimientos socioterritoriales como el MST. El MST es un movimiento socioterritorial altamente dinámico que ha evolucionado durante diferentes fases históricas, que podemos analizar mediante nuestros cuatro ejes.

Primero, la ocupación y la lucha por la tierra ha sido una estrategia central de las familias campesinas que reclaman una reforma agraria en Brasil, con el territorio como el objeto central de la disputa. El MST tiene sus raíces en los estados de Rio Grande do Sul, Paraná, Santa Catarina, São Paulo y Mato Grosso do Sul, donde miles de familias se apropiaron del espacio y comenzaron a transformar la cuestión agraria brasileña. Formalmente, se fundó en 1984. La lucha por la tierra se centra en la estrategia de la conquista, o recuperación, de una fracción del territorio en el que se reproducen las condiciones básicas para la existencia del campesinado. En el curso de la movilización, las luchas del MST por la tierra se territorializaron en varios estados de Brasil (Fernandes, 2000a; Wolford, 2010). A medida que las familias conquistaban con éxito fracciones de territorio, creaban espacios de socialización política con los cuales organizaban más familias para ocupaciones de tierras, con lo que multiplicaban los espacios de lucha y resistencia a través de más territorios campesinos. Por medio de otras ocupaciones de tierras, el MST se consolidó y se territorializó en diez estados brasileños hacia la década de 1990.

Segundo, el MST desarrolló su identidad explícitamente en relación con su carencia de tierra y con su estrategia de ocupaciones y conquistas de fracciones de territorio. El acto de apropiarse políticamente del espacio, convertir la tierra en territorio campesino y luego territorializarse en todo Brasil ha definido el movimiento y le ha dado su raison d'être (Fernandes y Stedile, 1999). También ha permitido la supervivencia y reproducción del modo de vida campesino. Cortar el alambrado se convirtió en la imagen que representaba su historia porque cada corte de cerco tenía el potencial de convertirse en un territorio conquistado (Bradford y Rocha, 2002). La tierra ocupada genera nuevas subjetividades, nacidas del espacio-tiempo de lucha. En el curso de la movilización, las ocupaciones de tierras a menudo se acompañan con ocupaciones de edificios públicos y manifestaciones callejeras. Estas últimas son especialmente importantes para generar una imagen global del MST y forman parte importante de la "mística" del movimiento: actos colectivos o rituales por los cuales se forjan el sentido común y la identidad. La transformación de la realidad sólo es posible con la creación de nuevos espacios en los que las personas se encuentran y materializan sus objetivos. Las ocupaciones no son sólo territorios de lucha y resistencia, sino también espacios de encuentro en los cuales las familias comparten sus intenciones, sus metas, sus deseos.

En tercer lugar, el MST moviliza las ocupaciones de la tierra como espacios de socialización política. Las familias suelen comenzar con la creación de un espacio comunicativo, donde se encuentran y se identifican como campesinos en la pobreza, expropiados por la territorialización de la agricultura capitalista. Estos encuentros pronto desarrollan conocimientos sobre sus trayectorias y generan una conciencia sobre la necesidad de cambiar el curso de su vida. Las ocupaciones crean espacios interactivos que profundizan el entendimiento de las realidades de las familias y les permiten tomar decisiones como protagonistas de su propia historia, lo que forma la base para crear territorios de lucha y resistencia. Hay un cambio cualitativo a medida que las familias comienzan a mirar hacia afuera y hacen pública la lucha que están construyendo; a la vez, conquistan y producen otros territorios. Las familias campesinas, anteriormente expropiadas y desterritorializadas por ganaderos o corporaciones, ahora están organizadas dentro de un movimiento socioterritorial, ocupan fracciones de los territorios de la clase propietaria y capitalista, desafían la desigualdad y transforman sus realidades (Carter, 2015). 
En cuarto lugar, el crecimiento del MST requería una estructura organizativa a nivel nacional para abordar diversos problemas, como la producción agrícola, infraestructura, educación, salud, comercio, industria, tecnología, entre otros. Al igual que otros movimientos socioterritoriales, el MST creó sus propias instituciones, como centros de investigación, escuelas, tiendas cooperativas, entre otras, para reproducirse en el espacio y el tiempo. Al hacerlo, el MST comenzó a actuar en múltiples dimensiones (social, económica, política, cultural y ambiental) del territorio. Al mismo tiempo, amplió e internacionalizó sus actividades. Desde 1991, ha ayudado a crear Vía Campesina, una red mundial de movimientos campesinos, y ha participado activamente en ella. Y se ha territorializado en 24 de los estados de Brasil, con lo que se convirtió efectivamente en un movimiento nacional. Sin embargo, ser un movimiento socioterritorial campesino, luchar por la tierra y por el desarrollo de los modos de vida campesinos implica no sólo la creciente territorialización del movimiento sino también saber cómo transformar la lucha en políticas concretas. En tal sentido, ha buscado forjar relaciones con los partidos políticos y los actores gubernamentales. Sin embargo, hasta el día de hoy el MST ha mantenido su principio de autonomía con respecto al Estado y los partidos políticos, y ha creado sus propias coyunturas mediante alianzas políticas de izquierda. Por ejemplo, al concebir alianzas con otros movimientos socioterritoriales (vinculados a Vía Campesina) y los gobiernos, el MST ha movilizado con éxito la agroecología como un tema clave de la producción de alimentos en Brasil, con énfasis en el tema de la soberanía alimentaria. Después de casi cuatro décadas, el MST se ha convertido en una referencia e inspiración para los movimientos socioterritoriales en diversos contextos urbanos y rurales en todo el mundo.

El MST es uno de los ejemplos más claros de un movimiento socioterritorial para el cual la apropiación del espacio es fundamental para su estrategia de conquista y transformación de fracciones de territorios en todo Brasil, y para, por medio de alianzas transnacionales, apoyar y alentar una mayor movilización en todo el mundo. Al transformar las relaciones socioespaciales mediante las ocupaciones de tierras, el MST ha presionado al Estado para que transforme la situación agraria en todo el país, y simultáneamente ha luchado por el territorio de Brasil. La fuerza del movimiento socioterritorial se ha basado en su estrategia, identidad y prácticas de socialización política, todas las cuales están enraizadas en sus territorios de lucha. Al mismo tiempo, la fuerza del movimiento ha generado desafíos. Todo proceso de territorialización implica un proceso de desterritorialización y, al ocupar los territorios de los terratenientes y corporaciones para reclamar tierras y viviendas, las familias han sido perseguidas y muchos líderes de movimientos han sido arrestados y asesinados por hombres armados al mando de grandes terratenientes, corporaciones o políticos. Los movimientos socioterritoriales tienen la capacidad de representarse a sí mismos y esto puede generar inspiración y solidaridad en todo el mundo, así como una fuerte persecución de los actores políticos cuyos proyectos han sido desterritorializados por las movilizaciones.

\section{Argentina: la Organización Barrial Tupac Amaru (OBTa)}

A comienzos del siglo XXI los movimientos sociales urbanos, ligados a problemáticas de empleo y pobreza, cobraron una centralidad inédita en la Argentina. Los llamados movimientos piqueteros, movimientos de fábricas recuperadas y asambleas ciudadanas se multiplicaron a lo largo y ancho del país.

En la norteña provincia de Jujuy, parte de una región con un históricamente precario desarrollo económico y altísimos índices de pobreza, nacía en 1999 la Organización Barrial Tupac Amaru (OBTA) (Battezzati, 2014; Manzano, 2015; Gaona, 2016).

La OBTA se conforma, tal como su nombre lo indica, como organización barrial para responder colectivamente a diversas demandas básicas de un amplio sector de la población jujeña más desprotegida: en un primer momento, se ocupó de peticionar por bolsones de comida y organizar copas de leche en los barrios periféricos de la ciudad capital de la provincia, San Salvador de Jujuy. Luego, comenzó a delinear estrategias para resolver el problema generalizado de acceso al empleo, en un contexto de elevada desocupación, heredero 
de las medidas neoliberales implementadas en todo el país desde mediados de la década del setenta y con intensidad inusitada durante la década del noventa.

En diciembre de 2001 se produjo la crisis conocida como el "argentinazo" y el posterior cambio de gobierno en 2003, con el triunfo de Néstor Kirchner como presidente de la República. El nuevo gobierno inauguró o revitalizó diferentes líneas de financiamiento en políticas públicas para paliar la trágica situación social, junto con un reposicionamiento político e ideológico que tendía a acercar posiciones, crear alianzas o coordinar con algunas de las muchas organizaciones sociales protagonistas del período (Boyanovsky Bazán, 2010). La OBTA era una de ellas y pronto pasó a conformar una alianza bastante sólida con el gobierno nacional: se adaptó al modelo cooperativo y comenzó a recibir financiamiento para la construcción de viviendas sociales, bajo el Programa de Emergencia Habitacional (PEH).

El exitoso desempeño de la organización para construir viviendas en un plazo y con un costo menores a los esperados y exigidos se tradujo en un crecimiento exponencial y sostenido de la OBTA en los siguientes doce años. La organización logró una disciplina de trabajo y crecimiento impactantes que se tradujo en la construcción de territorios propios: barrios de vivienda con escuelas, centros de salud, fábricas, polideportivos, parques acuáticos y temáticos, cines, centros culturales, radios populares, etc. Todas eran instituciones en las cuales se asentaron miles de puestos de trabajo que, a su vez, se inscribieron en un "dispositivo de bienestar social" (Manzano, 2015, p. 26), dispositivo que tiene un fundamento territorial.

El ejemplo más paradigmático de este proceso es el Barrio de la Tupac en Alto Comedero, en San Salvador de Jujuy, donde la organización construyó 3000 viviendas. Es un claro proceso de territorialización mediante el cual la organización se apropia de un territorio urbano, determina su uso, sus reglas, sus posibilidades y construye identidad, poder y estrategia política a partir de allí.

En primer lugar, el territorio que construye la organización a partir de su entramado barrial no se constriñe solo a éste; por el contrario, a raíz de la capacidad de movilización de personas, recursos, soluciones y símbolos que la organización posee, acumula a nivel provincial un lugar central como sujeto político de activación y resolución de demandas que se concentran en los sectores populares informales, pero que también confluye con otros actores, como trabajadores formales y medianos empresarios.

Su principal estrategia de visibilización se concibe a partir del territorio construido para que los miembros de la Tupac puedan vivir y cubrir sus necesidades. En y desde este nuevo territorio, reproducen modos de convivir, distribuir y pautar los intercambios, ya sea en el campo de la educación, del trabajo, de la salud o de la cultura.

En todos los espacios de la organización los "cómo" y los "cuándo" de las actividades y sus reglas se construyen colectivamente y con pautas propias: todos los miembros de la Tupac participan de alguna copa de leche al afiliarse a la organización. Con esta deben colaborar con trabajo voluntario o con cualquier otro tipo de ayuda. Todos los niños y niñas acceden a la escuela, la secundaria y el terciario de manera gratuita y con trayectorias flexibles; todos los que necesiten asistencia médica pueden obtenerla de manera gratuita en los centros de atención de la organización. Los problemas, los conflictos y tensiones entre vecinos de los barrios de la Tupac o entre trabajadores de una misma cooperativa se resuelven en asamblea, que incorpora en la discusión al presidente de la cooperativa o, incluso, a máximos referentes de la organización.

La impronta de la OBTA se materializa en la manera como construyeron sus barrios y como han disputado el uso del suelo urbano: dejando a un lado la especulación inmobiliaria y el individualismo propio del esquema meritocrático liberal que sujeta las posibilidades de las personas a la capacidad de compra en el mercado. También ha facilitado reposicionamientos simbólicos, étnicos y clasistas al posibilitar que los indios y los pobres de Jujuy accedan simbólica y materialmente al uso del centro de la ciudad, al construir su sede en una de las calles mejor ubicadas de San Salvador. Es imposible no verlos: su sede, su colegio, su pileta de natación cubierta y climatizada, sus consultorios externos, su radio comunitaria se encuentran en el medio de la ciudad capital, entre transeúntes de clase media, profesionales, políticos y miembros de la "alta" sociedad jujeña. 
En segundo lugar, la identidad de la organización se amalgama en esta concepción territorial: son tupaqueros porque comparten un espacio propio simbólico y material: comparten una forma de concepción del trabajo, de la militancia, de la convivencia barrial, del modelo de ciudad. Comenzaron a construir otra subjetividad, anclada en la cultura del trabajo, la responsabilidad y la solidaridad. También, nutrida de una fuerte disciplina y bajo una organización notablemente verticalista en la cual, si bien las principales decisiones y líneas de acción se definen en asambleas, estas funcionan en la práctica como escenarios un tanto prefabricados, donde la voz primordial es la de las personas con fuertes liderazgos.

Estas nuevas subjetividades insurgentes y transformadoras se encuentran claramente asociadas a una forma de liderazgo territorialmente situado: Milagro Sala, una mujer jujeña, pobre, india, negra, que pudo desde esa identidad individual construir una identidad colectiva a su imagen, anclada fuertemente en su tierra. Quizás este es uno de los elementos que permitan explicar la trabajosa replicabilidad de la experiencia de Jujuy en otras provincias del país.

En tercer lugar, derivado de (y reforzando) lo anterior, el territorio en la OBTA puede concebirse como el dispositivo de producción y reproducción de procesos de socialización política. Los hombres y mujeres, adultos y jóvenes que se han incorporado a la Tupac no sólo han encontrado la resolución de muchas de sus demandas (de vivienda, de salud, educativas, laborales, culturales) sino que también han sabido construir valores, afectos, códigos comunes de comprensión del mundo, de tramitación de diferencias y disputas.

Se trata de nuevas relaciones sociales, nuevos valores y nuevas mediaciones para concebirse como un nosotros político y potente para la acción colectiva extracotidiana, bajo grandes movilizaciones y actos públicos. Pero también en actos y situaciones cotidianos de convivencia y construcción día a día de la organización en sus barrios, en sus copas de leche, en sus fábricas y escuelas.

Frente a un contexto hostil, frente al mercado económico y político dominante que sistemáticamente los invisibiliza como sujetos de derecho, que los denigraba como sujetos laborales y que los silenciaba como sujetos políticos, la posibilidad de la construcción política abierta desde la propia agencia puede ser considerada el primer gran paso para una nueva subjetividad insurgente y una socialización política transformadora en un territorio redefinido y construido en disputa con esos otros territorios de la política, de la economía y de la sociedad jujeña capitalista, blanca y urbana.

En cuarto y último lugar, referenciamos el proceso de institucionalización formal que protagonizó la OBTA a partir de 2013 con la creación de un partido político como herramienta electoral, el Frente Unidos y Organizados ( $\mathrm{FUyO}$ ). Este último eje puede ser considerado central, puesto que propone el tránsito del territorio político propio, producido, apropiado y controlado, al territorio político de las instituciones dominantes en el régimen político poliárquico. Del territorio de las movilizaciones, del poder popular, de la ocupación de las calles y plazas, de la construcción de barrios, de escuelas y de fábricas con reglas de funcionamiento y pautas de intercambio construidos desde la propia organización, al espacio de la representación electoralista, con su lógica y sus reglas propias. En otro trabajo se analiza el desempeño electoral del FUyO en las contiendas electorales de 2013 y 2015 (Torres, 2019); lo que interesa destacar aquí es que la base de legitimación y acumulación política que se expresó en el $\mathrm{FUyO}$ provino del desarrollo de la militancia territorial de la Tupac y que este proceso de institucionalización formal dependía por completo del "éxito" del territorio propio. Cuando este último comenzó a ser cuestionado y erosionado, sucedió lo mismo con la institución partidaria.

Esta última cuestión es central para sopesar la potencialidad (y en ocasiones, inevitabilidad) de la institucionalización de prácticas, modalidades y territorialidades de los movimientos sociales. ¿No es el territorio construido por estos movimientos una forma institucional (quizás no formal) que provee durabilidad y solidez a construcciones colectivas transformadoras? Esa forma institucional territorializada no deja, evidentemente, de tener un signo político definitorio en términos de relaciones de poder que lo vuelven, entonces, contingente y cambiante. 


\section{INGLATERRA: OCCUPY LONDON}

En 2011 se extendieron por cientos de ciudades de todo el mundo movimientos sociales que se definieron por el hecho de ocupar el espacio público y que, en muchos casos, sufrieron una crisis de existencia al abandonar sus ocupaciones (Castells, 2012; Brown, Feigenbaum, Frenzel y McCurdy, 2017). Occupy London proporciona un estudio de caso interesante, dado que produjo uno de los campamentos de protesta que se extendió por más tiempo en 2011, con la ocupación principal en el patio de la icónica Catedral de San Pablo (frente a la Bolsa de Valores en el distrito financiero de Londres) durante cuatro meses y medio, y una ocupación posterior más pequeña en la cercana plaza Finsbury, que duró ocho meses, junto a numerosas ocupaciones ocasionales de edificios. La ocupación física del espacio urbano, movilizada en gran medida a través de las redes sociales como Facebook y mediante eventuales reuniones públicas, fue el acto constitutivo de Occupy London; y su capacidad para continuar "manteniendo" el espacio fue fundamental para su estrategia política. Cuando los activistas se reunieron en las escaleras de la Catedral de San Pablo el 15 de octubre de 2011, fecha de la convocatoria lanzada por los indignados españoles para realizar ocupaciones en todo el mundo, apareció un sistema portátil de sonido y se realizó la primera asamblea del movimiento, que tomó la decisión de ocupar la tierra en la que estaban sentados. A partir de este momento, Occupy London se constituyó en y a través del espacio de ocupación, y tomó decisiones mediante asambleas dos veces al día. Sin embargo, a diferencia de los casos de movimientos anteriores descritos en este trabajo, se mantiene una tensión entre la apropiación del espacio como medio y como objeto de movilización que arroja dudas sobre si podemos categorizar Occupy como un movimiento socioterritorial. Consideremos a continuación los cuatro ejes seleccionados.

En primer lugar, la ocupación y la producción de territorio fueron fundamentales para la estrategia de Occupy London, en relación con múltiples propósitos. La ubicación del campamento, en un sitio de alto perfil y exposición, generó una amplia cobertura y atención por parte de los medios de comunicación, especialmente cuando, inesperadamente, el cuerpo directivo de la Catedral cayó en una crisis interna sobre cómo responder ante la ocupación. Si bien esto restó atención al "espacio objetivo" previsto (Sparke, 2012) de la Bolsa de Valores, abrió con éxito el debate público en el Reino Unido sobre las crecientes desigualdades, las injusticias del capitalismo neoliberal y el derecho de las personas de acceder a la ciudad y de ocuparla. Las ocupaciones pueden ser vehículos altamente efectivos para crear lo que Lefebvre (2013) denomina "espacios opuestos de representación", utilizando la visibilidad del territorio apropiado como medio de movilizar un mensaje político hacia el exterior. Además, para un pequeño grupo de ocupantes, la visibilidad y la presencia mediática de la ocupación proporcionaron cierta influencia para imponer a los objetivos ciertas demandas, sobre todo a la City of London Corporation: el organismo gobernante del distrito financiero de Londres, que ha sido criticado por su creación de un paraíso fiscal (Shaxson, 2012). En la práctica, tales esfuerzos se vieron limitados por la falta de voluntad de la Corporación y otras instituciones financieras para negociar y, lo más importante, por la hostilidad de la mayoría de los ocupantes para entrar en una política reivindicativa, en rechazo de la legitimidad de todas las formas de representación institucional, particularmente aquellas vinculadas al capital financiero. De cualquier modo, el territorio era, en muchos aspectos, un problema secundario para los ocupantes que luchaban por las estructuras e ideas en una escala geográfica indefinida, pero ciertamente más allá de la materialidad de la plaza.

Al mismo tiempo, sin embargo, el territorio era fundamental para la estrategia de prefigurar y experimentar con formas alternativas de organización social y política. Desde la educación, el gobierno, los medios de comunicación y la ecología, el campamento intentó no solo actuar como un lugar de resistencia, sino también proporcionar un modo alternativo de organización al neoliberalismo y a la democracia parlamentaria creando una "grieta" en el capitalismo (Holloway, 2011) o un "Territorio disidente" (Souza, 2015). Si bien esta territorialización prefigurativa de Occupy (Ince, 2012; Routledge, 2015) fue simbólicamente poderosa, también expone las principales limitaciones del movimiento. Al priorizar la práctica de territorialización en 
la forma del campamento de protesta, Occupy London marginó la construcción de redes extra-locales con movimientos en otros lugares por falta de interés y/o capacidad (Halvorsen, 2012). Además, aquellos que vieron el campamento de protesta como un fin en sí mismo tendían a fetichizar el territorio de la ocupación, lo que cegaba su visión estratégica. Como uno de los ocupantes, Paul, expresó: "Algunas personas se perdieron, los árboles les taparon el bosque, y todo lo que pudieron ver fue el campamento, y no vieron ningún propósito más amplio (...) así que ese fue uno de los problemas, había un grupo de personas para quienes el campamento era un fin en sí mismo" (Paul, militante de Occupy, entrevista realizada por Halvorsen 3/09/2012).

En segundo lugar, la identidad de los activistas estaba fuertemente vinculada a la apropiación del espacio, tanto en su nombre ("Ocupar", "ocupantes") como en la práctica. La ocupación y el movimiento se convirtieron en una unidad y muchas personas vieron sus vidas subsumidas en Occupy London. Esto se desarrolló a través de una dialéctica de, por un lado, la experiencia intensa y afectiva de la territorialización como un espacio de ruptura, que se describe como una alegría de "alto voltaje" y un "remolino" que absorbió a los ocupantes en la medida en que muchos abandonaron sus hogares, trabajos y seres queridos para participar, y, por otro lado, las prácticas cotidianas mundanas y continuas de dirigir un campamento. Esta dialéctica expuso contradicciones dentro del campamento, por la marginación del trabajo material de reproducción de divisiones de género y de clase, y aumentó la tendencia de los ocupantes a fetichizar el territorio de la ocupación, lo que limitó su capacidad para re-imaginar el movimiento más allá de la ocupación. Sin embargo, es importante destacar que la experiencia afectiva de la ocupación creó nuevas subjetividades políticas que permanecieron políticamente activas mucho después del desalojo.

En tercer lugar, la ocupación produjo un espacio de encuentro, o socialización política, que reunió a personas con diversas trayectorias de activismo y proporcionó un espacio comunicativo en el cual los activistas articularon visiones colectivas sobre otros mundos. Sara, que había luchado para encontrar grupos políticos con los que se sintiera cómoda, expresó: “(...) fue llegar a Occupy y de repente sentir que había tanta gente con la que estaba conectada, y que podía entender, y con la que podíamos comunicarnos. ¡Fue realmente impactante! ¡Porque no había encontrado eso durante mucho tiempo!” (Sara, militante de Occupy, entrevista realizada por Halvorsen 7/09/2012).

Para muchos activistas, el espacio de encuentro generó experiencias que les cambiaron la vida, de las cuales aprendieron nuevas posibilidades (en términos de lo que eran social y políticamente capaces), y conocieron una red de personas con la que continuaron organizándose políticamente años más tarde. La ubicación de la ocupación, asimismo, produjo encuentros con trabajadores financieros, empresarios locales y residentes, que también catalizaron nuevas identidades y articulaciones. Estos encuentros, aunque no siempre positivos, fueron a menudo generadores de nuevos entendimientos y provocaron la ruptura de los estereotipos preconcebidos del otro. Sin embargo, a diferencia del MST y la OBTA, los valores creados en estos encuentros se relacionaron de manera ambigua con el espacio apropiado y se orientaron hacia temas más amplios en torno al capitalismo, la regulación estatal y los problemas del espacio público urbano, lo que impone límites a nuestro análisis de Occupy como un movimiento socioterritorial.

Cuarto, la territorialización de Occupy London generó procesos iniciales de institucionalización. Por otro lado, la práctica de ocupar y el acto de reunirse y tomar decisiones en el campamento dos veces al día formaron la base de su institucionalización autónoma, o "poder constituyente" (Hardt y Negri, 2012). Una "declaración inicial", escrita en una asamblea el segundo día de ocupación, proporcionó un conjunto de principios fundadores para el movimiento (Occupy London, 2011). A pesar del potencial radical del movimiento, hubo pocos intentos de institucionalizarse más allá de la escala del campamento y con otras ocupaciones en todo el mundo. Por otro lado, Occupy London se enredó en instituciones externas, particularmente con el Estado británico, lo que llevó a los ocupantes a una batalla legal prolongada sobre su derecho a ocupar suelo considerado de la "vía pública". De esta manera, para negociar en la Corte Superior, los ocupantes se vieron obligados a internalizar ciertas lógicas territoriales basadas en el Estado, que incluían tener un único representante en el tribunal, la necesidad de una estructura clara, el ordenamiento de las tiendas de campaña 
(bajo el pretexto de la reglamentación contra incendios) y el reconocimiento de los propietarios legales de la tierra. Esto creó tensiones en el campamento, ya que algunos rechazaron abiertamente la legitimidad de reclamos territoriales externos, mientras que otros se inclinaron por la posibilidad de establecer nuevos precedentes legales sobre el derecho a ocupar tierras en protestas políticas.

Estos dilemas pronto se volvieron insignificantes a medida que se desarrollaba el inevitable desalojo del campamento a principios de 2012 y los ocupantes se vieron obligados a volver a imaginar el movimiento sin la materialidad de la ocupación, inicialmente representada en el eslogan "No se puede desalojar una idea". Esto expuso la debilidad de Occupy London como un movimiento socioterritorial. Luego del desalojo podemos rastrear los intentos iniciales de reterritorialización. Primero, y aun movilizándose bajo la bandera de "Occupy London", los ocupantes dirigieron su atención a los vecindarios como un sitio de organización política: participaron en campañas locales contra los recortes (por ejemplo, luchando contra el cierre de bibliotecas públicas) y unieron fuerzas con movimientos de ocupantes ilegales. Esto proporcionó mayores recursos al movimiento y también resolvió algunas de las tensiones causadas por la separación entre espacios de reproducción social y de activismo. Sin embargo, ello siguió siendo un foco marginal para la mayoría de los ocupantes y pronto se disipó. En segundo lugar, varios años después y ya no con la identidad de Occupy London, varios ocupantes han dirigido su atención a la institución revitalizada del partido laborista del Reino Unido en un intento por ingresar a la política electoral del partido. Esta estrategia fue particularmente evidente en el pasaje de muchos indignados al partido Podemos en España y abre vías para futuros análisis sobre la territorialización de los movimientos sociales dentro de las estructuras estatales y de partidos.

\section{Palabras finales}

Todos los movimientos sociales se movilizan en y a través del espacio, pero sólo en ciertos casos la apropiación del espacio demarcado en la búsqueda de proyectos políticos específicos es un objetivo central. Nuestro análisis ha demostrado dos fuertes ejemplos de movimientos socioterritoriales: el MST y la OBTA. Aunque Occupy London demostró varias tendencias propias de los movimientos socioterritoriales, el territorio fue un elemento secundario de su estrategia, o bien se fetichizó, en forma de un campo de protesta, en detrimento de los objetivos y metas del movimiento. Los movimientos socioterritoriales tienden a surgir en un intento de desterritorializar los proyectos políticos del territorio estatal moderno y sus modos de gobierno, y también la neoliberalización del espacio (la producción de lo que Lefebvre denomina espacio abstracto), y de reterritorializar un proyecto político alternativo. En este sentido, los movimientos socioterritoriales son una categoría global que toma forma en el contexto de lo que Santos (1996) denomina "configuraciones territoriales" particulares, capas históricas de espacios apropiados en el contexto de proyectos políticos en desarrollo. Los movimientos socioterritoriales se movilizan en diferentes contextos histórico-geográficos que ayudan a explicar parte de sus oportunidades y limitaciones, como se puede ver en los tres estudios de caso aquí desarrollados.

El MST se movilizó en el contexto de una distribución altamente desigual de la propiedad de la tierra, un legado de la oligarquía latifundista poscolonial de Brasil que históricamente ha excluido a la clase campesina. El hecho de que el Estado no haya implementado una reforma agraria significativa impulsó a los movimientos sociales a movilizarse directamente para reclamar el derecho a la tierra mediante la ocupación y la producción de territorio. La extensión de la territorialización del MST a través de Brasil y sus simpatías posteriores con elementos del Partido de los Trabajadores bajo la presidencia de Lula reforzaron el movimiento y facilitaron su institucionalización más allá de la escala de ocupaciones individuales. Sin embargo, más recientemente, la conjunción de intereses de latifundios y agronegocios, conocida como el "latifundio binomial", ha intensificado la concentración de tierras dentro de una nueva dinámica de acaparamiento de ellas que está altamente enredada con las fuerzas del capitalismo global. En este contexto, la movilización del MST puede entenderse junto con un crecimiento regional e incluso mundial de movimientos socioterritoriales que 
buscan resistir el neo-extractivismo y construir un espacio apropiado para proyectos político-económicos alternativos (Svampa, 2017). La creación de movimientos socioterritoriales es ineludible por la existencia del campesinado excluido brasileño y también por muchos movimientos sociales indígenas en América Latina cuya tierra ha sido amenazada directamente por las políticas neo-extractivistas, políticas que se desarrollaron durante el ciclo progresista de la región y se han intensificado a nuevos niveles bajo el actual giro a la derecha (Svampa, 2017).

El caso del OBTA es interesante, ya que surgió de la intersección entre la marginación postcolonial (y el "borramiento" en el caso de la Argentina) de los pueblos indígenas de la política latinoamericana y el rápido crecimiento de las clases trabajadoras urbanas excluidas durante la Argentina postindustrial, particularmente durante el gobierno neoliberal del presidente Menem (1989-1999). La producción de territorio urbano, en forma de barrios o "pequeña ciudad dentro de la ciudad", fue fundamental para la producción de nuevas subjetividades políticas y formas de organización política en Argentina durante los años 90 y principios de los 2000 (Delamata, 2005; Merklen, 2005; Svampa y Pereyra, 2003). Los movimientos socioterritoriales, como la OBTA, surgieron en una coyuntura histórica en la que los militantes identificaron explícitamente el territorio como una estrategia central para la supervivencia, a través de redes de ayuda mutua y solidaridad, y de resistencia, mediante la focalización en los poseedores de poderes clave, a menudo utilizando la táctica de cortes de ruta, que culminó con el derrocamiento del presidente de la Argentina en diciembre de 2001. Tras la elección de los gobiernos de centro-izquierda o progresistas de Néstor Kirchner y Cristina Fernández de Kirchner (2003-2015), algunos movimientos socioterritoriales vieron una oportunidad política desde abajo y también fueron seducidos desde arriba para volver a territorializar sus luchas dentro del Estado argentino, con la inclusión en iniciativas de formulación de políticas y estructuras de gobierno local (Natalucci et. al., 2013; Rossi, 2017). Esto fortaleció el poder de los movimientos socioterritoriales y, en algunos casos, socavó y redujo su autonomía para determinar cómo se estaban produciendo y gobernando los territorios locales. En este contexto, la elección del presidente de derecha Macri a fines de 2015 ha planteado un desafío profundo y quizás mortal a los movimientos socioterritoriales que han perdido sus alianzas estatales.

Finalmente, Occupy London surgió en el contexto de una crisis financiera mundial, que típicamente se manifestó localmente como una crisis de legitimidad de la democracia representativa, debido a las fallas de las clases políticas para responder a las necesidades de la mayoría de la población. La ocupación de las plazas fue, ante todo, un desafío a las ideas universales y abstractas que sustenta el capitalismo neoliberal y su apoyo implícito por parte de las instituciones políticas. Al mismo tiempo, sin embargo, las prácticas prefigurativas de territorialización se materializaron e hicieron visible la existencia de órdenes sociales, económicos y políticos alternativos, lo que dio a los movimientos la esperanza de que, a través de la autoorganización, otros mundos posibles podrían ser creados en el aquí y el ahora. Aunque simbólicamente importante en el contexto de la destrucción del espacio público urbano, la plaza no fue el principal objeto de discusión. Las identidades afectivas y los encuentros que se generan en el curso de la apropiación del espacio, sin embargo, llevaron a Occupy London a una fetichización del territorio del campamento como medio y como fin de la lucha. Esto puso una barrera real a la capacidad del movimiento para evolucionar hacia un movimiento socioterritorial de la manera que aconteció, por ejemplo, en España y el movimiento de indignados.

Hemos propuesto los movimientos socioterritoriales como una categoría de relevancia global, pero reconocemos su arraigo en los contextos locales y su particular fortaleza en América Latina. Al desarrollar y ampliar los conocimientos de los académicos norteamericanos y europeos sobre los movimientos sociales, los latinoamericanos han generado, durante las últimas tres décadas, un conjunto rico de teorías para dar sentido a cómo y por qué se produce la movilización de base (Rossi y Bülow, 2015). Una de las ideas centrales de esta literatura ha sido la centralidad del territorio para los movimientos sociales (Zibechi, 2007). En contra de la mentalidad epistémica que ha tendido a impregnar disciplinas como la geografía, sugerimos que los académicos anglófonos tienen mucho que aprender de los debates latinoamericanos y conceptos como el de movimientos socioterritoriales pueden proporcionar una mayor comprensión de los movimientos 
sociales en diversos contextos histórico-geográficos. A pesar de tener una clara presencia y potencial en América Latina durante las últimas tres décadas, no hay razón para limitar nuestro análisis a esta región. Es probable que la neoliberalización del espacio urbano y la intensificación de las prácticas extractivistas (por ejemplo, el fracking) en las zonas rurales de todo el mundo proporcionen el impulso para la movilización del territorio como objeto de disputa por parte de diversos movimientos sociales. Cuando el intento de apropiarse del espacio para territorializar proyectos políticos alternativos se convierta en la lógica que guía la acción, podremos comenzar a hablar de movimientos socioterritoriales y comenzar a comprender los desafíos que enfrentan.

El movimiento socioterritorial es una categoría abierta y dinámica que no se ajusta a un modelo y está siempre estructurada por el contexto geográfico e histórico. Los cuatro ejes analizados indican una serie de oportunidades y desafíos que enfrentan los movimientos socioterritoriales, que pueden estar ocultos o marginados por la teoría de movimientos sociales o por un análisis de los movimientos socioespaciales. Nuestro objetivo no ha sido disminuir la relevancia e importancia de estos enfoques, sino más bien extenderlos y desarrollarlos. En este sentido, consideramos necesarios más análisis teóricos y empíricos de los movimientos socioterritoriales y sobre la relación entre el territorio y la política de base.

\section{ReFERENCIAS}

Agnew, J. y Oslender, U. (2013). Territorialities, Sovereignty in Dispute: Empirical Lessons from Latin America. En W. Nicholls, B. Miller B. y J. Beaumont (Eds.), Spaces of Contention: Spatialities and Social Movements (pp. 121-140). Farnham: Ashgate.

Battezzati, S. (2014). La Tupac Amaru: movilización, organización interna y alianza con el kirchnerismo (2003-2011). Población \& Sociedad 21(1), 5-32. Recuperado de https://ppct.caicyt.gov.ar/index.php/pys/article/download/ $1879 / \mathrm{pdf}$

Boyanovsky Bazán, C. (2010). El aluvión del piquete al gobierno. Movimientos sociales y kirchnerismo. Buenos Aires: Sudamericana.

Bradford, S. y Rocha, J. (2002). Cutting the wire: The story of the landless movement in Brazil. Londres: Latin America Bureau.

Brown, G., Feigenbaum, A., Frenzel, F. y McCurdy, P. (Eds.) (2017). Protest Camps in International Context: Spaces, Infrastructures and Media of Resistance. Bristol: Policy Press.

Carter, M. (Ed.) (2015). Challenging Social Inequality. Durham: Duke University Press.

Castells, M. (2012). Redes de indignación y esperanza: los movimientos sociales en la era de Internet. Madrid: Alianza Editorial.

Cervio, A. L. y Vergara, G. (2017). Segregación socio-espacial, conflictos y sensibilidades: disputas por la movilidad y el desplazamiento en la ciudad de Córdoba, Argentina. Aposta. Revista de Ciencias Sociales, 74, 111-144. Recuperado de http://apostadigital.com/revistav3/hemeroteca/acervio.pdf

Delamata, G. (Ed.) (2005). Ciudadanía y territorio: Las relaciones politicas de las nuevas identidades sociales. Buenos Aires: Espacio.

Dinerstein, A. (2015). The Politics of Autonomy in Latin America. Basingstoke: Palgrave Macmillan.

Elden, S. (2013). The Birth of Territory. Londres: University of Chicago Press.

Fernandes, B. M. (2005). Movimentos socioterritoriais e movimentos socioespaciais: contribução teórica para uma leitura geográfica dos movimentos sociais. Revista Nera 8(6), 14-34.

Fernandes, B. M. (2000a). A Formação do MST no Brasil. Petrópolis: Editora Vozes.

Fernandes, B. M. (2000b). Movimento Social como Categoria Geográfica. Revista Terra Livre, 15, 59-85.

Fernandes, B. M. y Martin, J. Y. (2004). Movimento socioterritorial e "globalização": algumas reflexões a partir do caso do MST. Lutas Sociais v. 12. 
Fernandes, B. M. y Stedile, J. P. (1999). Brava gente: a trajetória do MST e a luta pela terra no Brasil. São Paulo: Editora Fundação Perseu Abramo.

Gaona, M. (2016). Experiencia, ciudad e identidad en torno a la organización barrial Tupac Amaru de San Salvador de Jujuy (tesis doctoral). Facultad de Periodismo y Comunicación Social, UNLP. Recuperado de http://sedici.un lp.edu.ar/handle/10915/52003

Girardi, E. et al. (2017). Relatório DATALUTA Brasil 2016. Presidente Prudente: NERA.

Glassman, J. (2002). "From Seattle (and Ubon) to Bangkok: The Scales of Resistance to Corporate Globalization", Environment and Planning D: Society and Space, 20(5), 513-533.

Grimson, A., Ferraudi, C. M. C. y Segura, R. (2009). La vida politica en los barrios populares de Buenos Aires. Buenos Aires: Prometeo.

Haesbaert, R. (2004). O mito da desterritorialização. Do "Fim dos Territorios" a multiterritorialidade. Rio de Janeiro: Bertrand Brasil.

Haesbaert, R. (2013) Del mito de la desterritorialización a la multiterritorialidad. Cultura y representaciones sociales, 8(15), 9-42. http://www.journals.unam.mx/index.php/crs/article/view/41590

Halvorsen, S. (2012). Beyond the Network? Occupy London and the Global Movement'. Social Movement Studies, $11(3-4), 427-433$.

Halvorsen, S. (2019). Decolonising Territory: dialogues with Latin American knowledges and grassroots politics. Progress in Human Geography, 43(5), 790-814.

Halvorsen, S., Mançano Fernandes, B. y Torres, F. V. (2019). Mobilizing Territory: Socio-territorial Movements in Comparative Perspective. Annals of the American Association of Geographers. Recuperado de https://doi.org/1 $0.1080 / 24694452.2018 .1549973$

Hardt, M. y Negri, A. (2012). Declaration. Recuperado de https://antonionegriinenglish.files.wordpress.com/2012/ 05/93152857-hardt-negri-declaration-2012.pdf

Hart, G. (2016). Relational comparison revisited: Marxist postcolonial geographies in practice. Progress in Human Geography online Recuperado de https://doi.org/10.1177/0309132516681388

Holloway, J. (2011). Agrietar el capitalismo: el hacer contra el trabajo. México: Benemérita Universidad de Puebla.

Ince, A. (2012). In the shell of the old: Anarchist geographies of territorialisation. Antipode 44(5), 1645-1666.

Juris, J. S. (2008). Networking Futures: The movements against corporate globalization. Durham: Duke University Press. Keith, M. y Pile, S. (Eds.) (1993). Place and the Politics of Identity. Londres: Routlegdge.

Lefebvre, H. (2013). La producción del espacio. Madrid: Capitán Swing.

Lopes de Souza, M. (1995). O território: sobre espaço e poder, autonomia e desenvolvimento. En I. Elias de Castro, P. Da Costa Gomes y R. Lobato Azevedo Corrêa (Coords.), Geografia: conceitos e temas (pp. 77-115). Rio de Janeiro: Bertrand Brasil.

Lopes de Souza, M. (2011). Autogestión, “autoplaneación”, autonomía: Actualidad y dificultades de las prácticas espaciales libertarias de los movimientos urbanos. En G. Calderón y E. León (Orgs.), Descubriendo la espacialidad social desde América Latina: reflexiones desde la geografia sobre el campo, la ciudad y el medio ambiente (pp. 53-90). Ciudad de México: Itaca.

Lopes de Souza, M (2015). Dos Espaços de Controle Aos Terriórios Dissidentes: Escritos de divulgação cientifica e análise política. Rio de Janeiro: Consequencia.

Manzano, V. (2015). Lugar, trabajo y bienestar: la organización barrial Tupac Amaru en clave de política relacional. Publicar, año XVIII n ${ }^{\circ}$ XIX, 9-35. Recuperado de https://ppct.caicyt.gov.ar/index.php/publicar/article/view/ $7844 / 7063$

Martin, D. G. (2003). Place-framing as place-making: constituting a neighbourhood for organizing and activism. Annals of the Association of American Geographers. 93(3), 730-750.

Massey, D. (1991). A Global Sense of Place. Marxism Today, junio, 24-29.

McAdam, D., Tarrow, S. y Tilly, C. (2005). Dinámica de la contienda politica. Barcelona: Hacer Editorial. 
Melucci, A. (1980). The new social movements: a theoretical approach. Social Science Information, 19(2), 199-226.

Merklen, D. (2005). Pobres ciudadanos: las clases populares en la era democrática (Argentina 1983-2003). Buenos Aires: Editorial Gorla.

Mignolo, W. D. (2000). Local Histories/Global Designs: Coloniality, Subaltern Knowledges, and Border Thinking. Oxford: Princeton University Press.

Miller, B. A. (2000). Geography and Social Movements: Comparing Antinuclear Activism in the Boston Area. Londres: University of Minnesota Press.

Natalucci, A. et. al. (2013). Territorios disputados: Movilización política y procesos de institucionalización en niveles locales de gobierno (Argentina, 2003-2011). Revista Mexicana de Análisis Politico y Administración Pública, II (2) $139-159$.

Nicholls, W. (2009). Place, networks, space: theorising the geographies of social movements. Transactions of the Institute of British Geographers, 34, 78-93.

Nicholls, W., Miller, B. y Beaumont, J. (Eds.) (2013). Spaces of Contention: Spatialities and Social Movements. Farnham: Ashgate.

O`Donnell, G. (1993). Estado, democratización y ciudadanía. Nueva Sociedad, N. ${ }^{\circ} 128$. Recuperado de https://nuso .org/articulo/estado-democratizacion-y-ciudadania/

Occupy London (2011). Initial statement. Recuperado de https://occupylondon.org.uk/about/statements/initial-st atement

Oslender, U. (2002). Espacio, lugar y movimientos sociales: hacia una espacialidad de resistencia. Scripta Nova VI (115). Recuperado de http://www.ub.es/geocrit/sn/sn-115.htm

Poma, A. y Gravante, T. (2017). Emociones, protesta y acción colectiva: estado del arte y avances. Aposta. Revista de Ciencias Sociales, 74, 32-62. Recuperado de http://apostadigital.com/revistav3/hemeroteca/apoma.pdf

Porto-Gonçalves, C. W. (2001). Geo-grafias. Movimientos sociales, nuevas territorialidades y sustentabilidad. México: Siglo XXI.

Raffestin, C. (1980). Pour une Geographie du Pouvoir. París: LITEC.

Reyes, A. (Eds.) (2012). Special Issue: Autonomy and Emancipation in Latin America. South Atlantic Quarterly, $111(1), 1-225$.

Robinson, J. (2016). Thinking cities through elsewhere: Comparative tactics for a more global urban studies. Progress in Human Geography, 40(1), 3-29.

Rossi, F. M. (2017). The Poor's Struggle for Political Incorporation: The Piquetero Movement in Argentina. Cambridge: Cambridge University Press.

Rossi, F.M. \& Bülow, M. (Eds.) (2015). Social movement dynamics: new perspectives on theory and research from Latin America. Farnham: Ashgate.

Routledge, P. (1993). Terrains of Resistance: Nonviolent social movements and the contestation of place in India. Westport Praeger.

Routledge, P. (2015). Territorialising movement: the politics of land occupation in Bangladesh. Transactions of the Institute for British Geographers, 40(4), 445-463.

Routledge, P., 2017. Space Invaders: Radical Geographies of Protest. Londres: Pluto.

Routledge, P. y Cumbers, A. (2009). Global Justice Networks: Geographies of Transnational Solidarity. Manchester: Palgrave Macmillan.

Santos, B. S. (2009). Una epistemología del Sur. México: CLACSO-Siglo XXI Editores.

Santos, M. (1996). A Natureza do Espaço. São Paulo: Hucitec.

Santos, R. E. dos (2011). Movimentos sociais e geografia: sobre a(s) espacialidade(s) da ac\#a\#o social. Rio de Janeiro: Conseque\#ncia.

Saquet, M. A. (2009). Por uma abordagem territorial. En A. M. Saquet y E. S. Sposito, Territórios e territorialidades: teorias, processos e conflitos (pp. 197-215). São Paulo: Expressão Popular. 
Schneider, S. y Peyré Tartaruga, I. (2006). Territorio y enfoque territorial: de las referencias cognitivas a los aportes aplicados al análisis de los procesos sociales rurales. En M. Manzanal, G. Neiman y M. Lattuada (eds.), Desarrollo rural: Organizaciones, instituciones y territorios (pp. 71-102). Buenos Aires: CICCUS.

Scribano, A. (2008). Cuerpo, conflicto y emociones en Argentina después del 2001. Revista Espacio Abierto,17, 205-230.

Shaxson, N. (2012). Treasure Islands: Tax Havens and the Men who Stole the World. Londres: Vintage.

Sitrin, M. (2012). Everyday Revolutions: Horizontalism and Autonomy in Argentina. Londres: Zed Books.

Sparke, M. (2012). From Global Dispossession to Local Repossession: Towards a Worldly Cultural Geography of Occupy Activism. En J. Winder y R. Schein (Eds.), The New Companion to Cultural Geography (pp. 387-408). Oxford: Wiley-Blackwell.

Stahler-Sholk, R., Vanden, H. E. y Becker, M. (Eds.) (2014). Rethinking Latin American social movements: radical action from below. Rowman and Littlefield.

Svampa, M. (2017). Del cambio de época al fin del ciclo: gobiernos progresistas, extractivismo y movimientos sociales en América Latina. Buenos Aires: Edhasa.

Svampa, M. y Pereyra, S. (2003). Entre la ruta y el barrio: La experiencia de las organizaciones piqueteras. Buenos Aires: Biblios.

Tarrow, S. (1997). El poder en movimiento. Movimientos sociales, acción colectiva y politica. Madrid: Alianza.

Tobío, O. (2010). Entre el Estado y los movimientos sociales: sobre la recreación de lo público en función de la planificación territorial. Scripta Nova, Vol. XIV, núm. 331 (43) Recuperado de http://www.ub.edu/geocrit/sn /sn-331/sn-331-43.htm

Tobío, O. (2014). El doble carácter de la territorialidad piquetera en el norte de la Argentina: una conceptualización. Actas del XIII Coloquio Internacional de geocrítica. Recuperado de http://www.ub.edu/geocrit/coloquio201 4/Omar\%20Tobio.pdf

Torres, F. V. (2016). Henri Lefebvre y el espacio social: aportes para analizar procesos de institucionalización de movimientos sociales en América Latina. Sociologias, Año 18, n 43, 240-270 Recuperado de http://www.seer. ufrgs.br/index.php/sociologias/article/view/56550

Torres, F. V. (2019). Proceso de territorialización de la Organización Barrial Tupac Amaru: cooperativas, barrio y política. Revista Estudios socioterritoriales, 25, e023., 1-17. Recuperado de https://doi.org/10.37838/unicen/es t.25-023

Wolford, W. (2010). This Land is Ours Now: Social Mobilization and the Meanings of Land in Brazil. Durham, Duke University Press.

Zibechi, R. (2006). Dispersar el poder. Buenos Aires: Tinta Limón.

Zibechi, R. (2007). Territorios en resistencia. Buenos Aires: Lavaca.

\section{Notas}

1 La idea de que el territorio es movilizado es la traducción de la expresión "territory is mobilized", incluida en nuestro trabajo previo en inglés Halvorsen, Fernandes y Torres (2019:3).

2 La palabra enredado o enredar es la traducción del término 'entangled' o 'entangle' utilizado en inglés por Sam Halvorsen (Halvorsen, 2019).

3 De acuerdo con Haesbaert, la noción de lugar en la literatura anglosajona tiene una definición y es aplicada de manera similar a la categoría territorio en la literatura latinoamericana. 